\title{
Transfer of lumbosacral load to iliac bones and legs
}

\section{Part 2: Loading of the sacroiliac joints when lifting in a stooped posture}

\author{
C J Snijders $P h D^{1}$, A Vleeming $P h D^{2}$, R Stoeckart $P h D^{2}$ \\ ${ }^{1}$ Department of Biomedical Physics and Technology, and ${ }^{2}$ Department of Anatomy, \\ Faculty of Medicine and Allied Health Sciences, Erasmus University Rotterdam, \\ The Netherlands
}

\begin{abstract}
Summary
We developed a biomechanical model of load transfer by the sacroiliac joints in relation to posture. A description is given of two ways in which the transfer of lumbar load to the pelvis in a stooped posture can take place. One way concerns ligament and muscle forces that act on the sacrum, raising the tendency of the sacrum to flex in relation to the hip bones. The other refers to ligament and muscle forces acting on the iliac crests, raising the tendency of the sacrum to shift in caudal direction in relation to the hip bones. Both loading modes deal with the self-bracing mechanism that comes into action to prevent shear in the sacroiliac joints.

When a person is lifting a load while in a stooped posture, the force raised by gravity acting in a plane perpendicular to the spine and the sacrum becomes of interest. In this situation a belt such as used by weight lifters may contribute to the stability of the sacroiliac joints. Verification of the biomechanical model is based on anatomical studies and on load application to human specimens. Magnetic resonance imaging pictures have been taken to verify geometry in vivo.
\end{abstract}

\section{Relevance}

When performing a lifting task, muscle groups must act in concert. This pattern of muscle activities could contribute to the stability of the sacroiliac joints. Insight into these interrelations may supplement training programmes for muscle strength and coordination in the prevention and treatment of low back injuries.

Key words: Biomechanics, sacroiliac joint, lumbodorsal fascia, abdominal muscle, weight lifter

\section{Introduction}

The largest muscles of the human body attach to the pelvis, resulting in considerable load transfer. In principle every force generated by back muscles that acts directly or indirectly on the spine and on the iliac bones contributes to the load to be transferred by the sacroiliac (SI) joints.

The following discussion will be restricted to

Received: 10 April 1992

Accepted: 1 January 1993

Correspondence and reprint requests to: $\mathrm{C} \mathrm{J}$ Snijders, Erasmus University Rotterdam, Faculty of Medicine and Allied Health Sciences, PO Box 1738, 3000 DR Rotterdam, The Netherlands strenuous exertions which may be expected when a subject is lifting a load while in a forward bent posture. For stooped postures large intradiscal pressures in the lumbar area are reported ${ }^{1}$. Sophisticated models have been developed to estimate the forces in the spine and in the muscles of the trunk which are active in different postures $^{2-4}$. The concept of load transfer by the thoracolumbar fascia was introduced ${ }^{5}$; this fascia has a large lever arm with respect to the spine, resulting in lower calculated values of disc compression. Whereas the literature on the biomechanics of the thoracolumbar spine - including stress analysis ${ }^{\hat{6}}$ - has made great progress, little attention has been paid to the biomechanics of the pelvis. Studies of the pelvis that include forces are limited ${ }^{7.8}$ and do not deal with problems of SI joint stability in relation to posture. 
Therefore we have developed a biomechanical model of load transfer from the spinc to the pelvis and legs when lifting in a stooped posture.

\section{Posture and load on the sacrum}

\section{Forward bending}

In part 1 of this article the load on the sacrum was assumed to be equal to the weight of the suprasacral part of the body. However, due to muscle forces the corresponding SI joint reaction forces become much larger. In a forward bent posture the centre of gravity of the whole body is situated above the feet (see Figure 1b). To estimate the magnitude of the forces in the back, a cross-section is made in the lumbar area for the construction of a free-body diagram of the upper part of the body (Figure 1a). The weight of the part of the body above the cross-section is $F_{g}$ with its point of application near the armpit ${ }^{9}$. Equilibrium of forces in the vertical direction is obtained by the reaction force $F_{r g}$ in the spine which is equal in magnitude to $F_{g}$. Both forces form a couple which acts clockwise with a moment $F_{g}$ a. To obtain equilibrium of moments, the resultant back muscle force $\left(F_{s}\right)$ and its reaction force in the spine $\left(F_{r s}\right)$ form an anticlockwise moment with lever arm $b$. This simplified model is often used ${ }^{10-12}$ to elucidate the heavy load on the lumbar spine which is the consequence of the large ratio $a / b$ in a forward bent position. In this study we consistently draw the forces from muscles, ligaments and gravity $\left(F_{i}\right)$ together with their reaction forces $\left(F_{r i}\right)$ in the respective joint. This yields a configuration of couples of forces giving a clear distinction of each contribution to joint load. Moreover, in the free-body diagrams the contribution of moments in cross-sections due to deformation is not included for ease of survey (intrinsic joint stability). The free-body diagram of the upper part of the body is the first step leading to the analysis

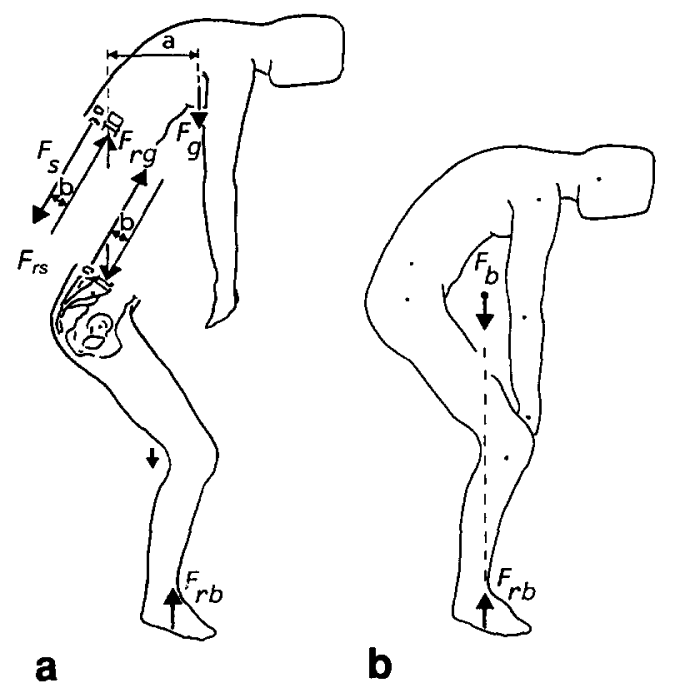

Figure 1. Bending forward is accompanied by considerable forces in cross-sections at lumbar levels. The pelvis is the next segment in this force stream.

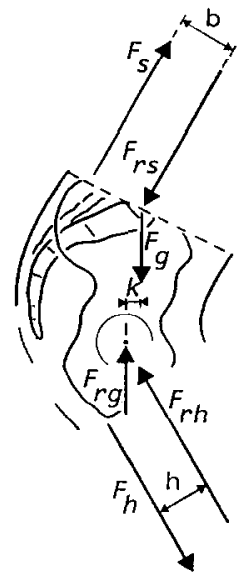

a b

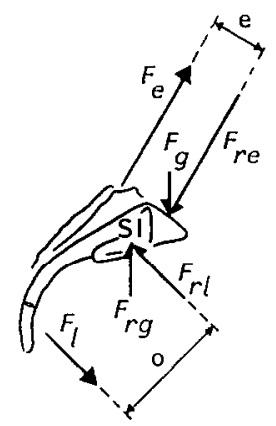

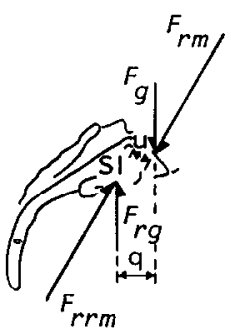

C
Figure 2. a, Equilibrium of the pelvis with reference to the posture in Figure 1. b, Equilibrium of the sacrum. Here it is assumed that back muscles $\left(F_{e}\right)$ are attached to the sacrum. The ligament and/or muscle force $\left(F_{j}\right)$ provide for equilibrium of moments of force. c, Equilibrium of the sacrum. Here it is assumed that forces of back muscles, abdominal muscles, and part of lumbar dorsal fascia $\left(F_{m}\right)$ act on the ilium. Their reaction force $F_{r m}$ in the spine must be transferred by the SI joints into $F_{r r m}$.

of the equilibrium of the part of the body below the lower lumbar cross-section (Figure 1a). So in this example, the load on the sacrum will be approximately five times the weight of the upper part of the body.

A free-body diagram of the pelvis (Figure $2 a$ ) can be based on cross-sections at the lower lumbar level (see Figure 1a) and at the hip joint. To rcach an cquilibrium of moments the action of muscles $\left(F_{h}\right)$ at the dorsal side of the upper leg (gluteus maximus muscles, hamstrings) is required. Figure $2 b$ and $c$ give a schematic representation of the equilibrium of the sacrum. First the weight of the upper part of the body $\left(F_{g}\right)$ is carried by the SI joints $\left(F_{r g}\right)$. Next the resultant back muscle force $F_{s}$ will be split into two parts. One part of this bifurcation refers to the forces acting on the sacrum, the other part to the forces acting on the iliac crests. In Figure $2 b$ a large moment of force will be the result of the tensile force from the back muscles (part of erector spinae) which are attached to the sacrum $\left(F_{e}\right)$ and its reaction force on the sacrum $\left(F_{r e}\right)$. Because $F_{e}$ and $F_{r e}$ are equal and have opposite directions, the SI joint does not 'feel' both. In this free-body diagram of the sacrum, equilibrium of moments requires filling in of a counterclockwise moment of force. This leads to the introduction of the force $F_{l}$ from the sacrotuberous ligament and sacrospinous ligament and its reaction force $F_{r l}$ in the SI joint. Because these ligaments are attached to the ilium, their tension will counteract flexion of the sacrum with respect to the pelvic ring. This mechanism becomes particularly interesting when we study the simultaneous action of the biceps femoris muscles in Figure 2a.

In an anatomical study ${ }^{13}$ it was demonstrated that in certain individuals the biceps femoris muscles are directly connected with the sacrotuberous ligament, 
which enables them to support the equilibrium according to Figure 2b. Even without clear tendon connections, force transfer between the biceps femoris muscles and sacrotuberous ligaments occurred, probably due to fascial connections. In addition, the pelvis-leg flexion angle influences the amount of force transfer between ligament and tendon. Fibres from the thoracolumbar fascia that are attached to the sacrum are also connected with the sacrotuberous ligaments. This means that the tendency to flexion of the sacrum in relation to the hip bones can simultaneously be counteracted via the thoracolumbar fascia.

The free-body diagram of the sacrum will be completely different in the situation where back muscle forces $\left(F_{m}\right)$ are transferred to the iliac crests directly, or indirectly by means of the lumbar dorsal fascia. This would lead to Figure $2 \mathrm{c}$, which shows disappearance of the moment with lever arm $e$ produced by $F_{e}$ and $F_{r e}$. Now, however, the SI joints must produce the reaction force $F_{r r m}$ which counteracts $F_{r m}$.

From the previous free-body diagrams it is attempted to isolate the different contributions to the complex loading situation. At present the contribution of each structure to the total load transfer is unknown, and these contributions will be different in different postures. Furthermore, it must be emphasized that accurate data about the geometry are not yet available. In Figure 2c, for example, the moments of force about the SI joint would be reduced by decreasing the lever arms $u$ and $q$. Here it can be speculated that the moment $F_{r m} \cdot u$ can become anticlockwise, especially with lumbar kyphosis.

\section{Bifurcation of the resultant back muscle force}

The model on the bifurcation, as introduced before,

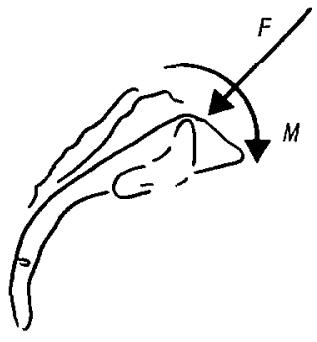

a

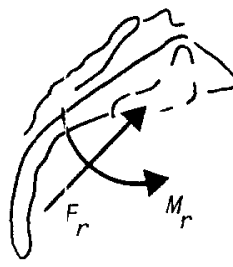

b
Figure 3. a, Bifurcation of the resultant back muscle force acting on the pelvis ( $F_{s}$ in Figure $2 a$ ) into $F_{e}$ (Figure $2 b$ ) and $F_{m}$ (Figure $2 c$ ) results in two loading modes of the sacrum. The $\mathrm{M}$ load produces a moment of force about the SI joint and results from back muscles and parts of the lumbodorsal fascia that are attached to the sacrum. The F load concerns a force in axial direction to the sacrum and results from all muscles, fascia and ligaments that act between iliac crests and upper body. b. Reaction of the SI joints in the transfer of both loading modes. The sacrotuberous and sacrospinal ligaments can contribute to the reaction moment $\left(M_{r}\right)$ which counteracts flexion of the sacrum in relation to the hip bones. The joint reaction force $\left(F_{r}\right)$ can be produced with the help of the self-bracing mechanism which is based on the model of the pelvic arch.

has far-reaching consequences for the understanding of load transfer by the SI joints. The consequences can be summarized with the help of Figure 3. The suprasacral muscles attached to the sacrum and tension in parts of the lumbodorsal fascia acting on the sacrum produce a moment of force about the SI joint, indicated with the curved arrow. This we call the $\mathrm{M}$ load. Muscle forces acting on the iliac crest directly, or indirectly via parts of the lumbodorsal fascia, produce a force axial to the

Table 1. Loads imposed on the SI joints (action) listed with possible contributions to prevent dislocation (reaction). It is realized that e.g. the erector spinae muscle and the lumbodorsal fascia have connections with the sacrum and with the iliac bones. Here, however, we restrict ourselves to the major contributions expected on the basis of the biomechanical model

Fload MLoad

\section{Action}

Lumbodorsal fascia

M. quadratus lumborum

M. obliquus abdominis

$M$ rectus abdominis

M. latissimus dorsi

M. psoas major

\section{Reaction}

M. gluteus maximus

$M$. biceps femoris in relation to sacrotuberous ligament

M. piriformis

$M$. coccygeus

Friction coefficient

Grooves and ridges

Interosseous and dorsal SI joint ligaments; sacrotuberous and sacrospinal ligaments Pelvic belt

MM. obliquus and transversus abdominis

Weight-lifter belt
Lumbodorsal fascia

M. erector spinae

Lever arm of $F$ load

M. gluteus maximus

$M$. biceps femoris in relation to sacrotuberous ligament Sacrospinal ligament

lliolumbar ligament

Twisted shape of joint

Friction coefficient

Grooves and ridges

Interosseous and dorsal SI joint ligaments;

sacrotuberous and sacrospinal ligaments

Pelvic belt 


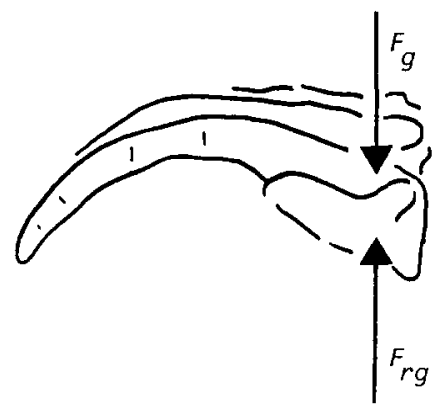

Figure 4. When the sacrum assumes a more or less horizontal position, the load consisting of the weight of the upper part of the body and the lifted object $\left(F_{g}\right)$ acts perpendicular to the spine. To provide for a joint reaction force $F_{r g}$ transverse to the sacrum, a self-bracing mechanism can come into action in this plane by means of the transverse and oblique abdominal muscles or by means of a belt such as used by weight lifters.
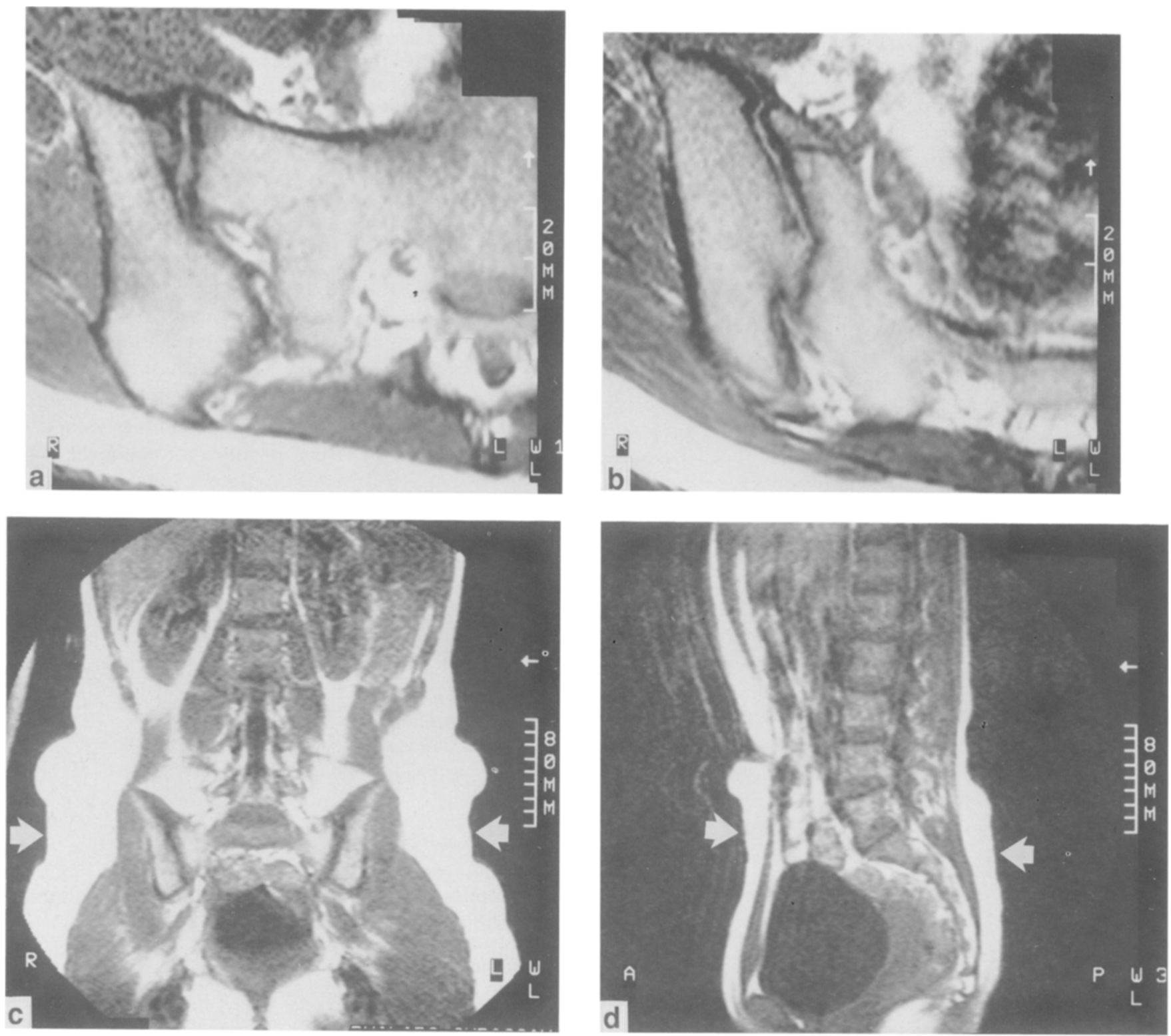

Figure 5. MRI picture of the SI joints, female age 23, body height $178 \mathrm{~cm}$ and body weight $640 \mathrm{~N}$. a, Transverse slice taken at the cranial side of the SI joint, and $\mathbf{b}$, transverse slice taken caudally. Comparison of both slices indicates a slightly twisted form of the joint surfaces. c, Coronal slice, and d, sagittal slice showing that the level of the cranial side of the SI joints coincides with the level of a belt used by weight lifters. The arrows point to the impression of the belt, which has no metal buckle. 
section (Figures 1 and 2), and contributes to both the $\mathrm{F}$ load and the $\mathrm{M}$ load. The force generated by gravity can be resolved in a component in longitudinal direction of the spine and sacrum, and one perpendicular to it. The latter becomes of special interest when lifting in a stooped posturc (Figure 4). The total weight of a lifted object and the upper part of the body $\left(F_{g}\right)$ can be considerable, and is directed perpendicular to the longitudinal axis of the sacrum. A self-bracing effect analogous to Figure 4 in part 1 would be helpful to warrant SI joint stability. In this plane of loading, however, other structures must produce the ligament or muscle force $\left(F_{l}\right)$, which is a key point in the model. In this posture the additional force needed can be introduced by the action of the transverse and oblique abdominal muscles in particular.

Weight lifters often use a special belt. Its mechanism is explained by the extra support it gives to the back by enlarging intra-abdominal pressure ${ }^{14.15}$. Often these belts are dorsally wide, but narrow at the ventral side, and are put on forcefully around the cranial side of the iliac crests. We hypothesize that the belt can help self-bracing of the SI joint when used in a stooped posture. To further analyse the position of the belt in relation to the SI joint we have taken MRI pictures (Figure 5). The magnetic resonance imaging (MRI) transverse slices of the SI joints at the cranial side (Figure 5a) and at the caudal side (Figure 5b) show different wedge angles. The wedge at the caudal side is ventrally more open than at the cranial side. This illustrates that at the cranial side of the SI joint the wedge angle is more appropriate for the loading mode in Figure 4. Therefore the bracing effect of a belt should be evoked in stooped postures at this level. Figures $5 \mathrm{c}$ and $\mathrm{d}$ show a cranial position of the belt (see arrows at the impressions from the belt). At a more cranial position the belt would slide from the iliac crests and would become slack.

\section{Curl-up}

To further illustrate the effect of posture on pelvic load, an abdominal muscle exercise is chosen. The free-body diagram of a seated person in Figure 6a shows that the seat supports the weight of the upper part of the body $\left(F_{r g}\right)$ and the weight of the pelvis, the legs, and the force on the foot $\left(F_{r b}\right)$. Abdominal muscles must be active to keep the upper part of the body in position. As a consequence the pelvis is loaded by a couple formed by $F_{m}$ and its reaction force in the spine $F_{r m}$. Equilibrium of moments requires a clockwise moment from muscles at the ventral side of the hip joint (couple formed by $F_{p}$ and $F_{r p}$ ). In Figure $6 \mathrm{~b}$ the free-body diagram of the sacrum is given schematically. It may be expected that exercises with the use of the greater psoas muscle involve greater SI joint forces, because its action can be compared with that of $F_{r m}$ in Figure 2c and will be added to $F_{r m}$ in Figure $6 \mathrm{~b}$. The magnitude of the forces is highly dependent on the respective lever arms that determine the moments of force.
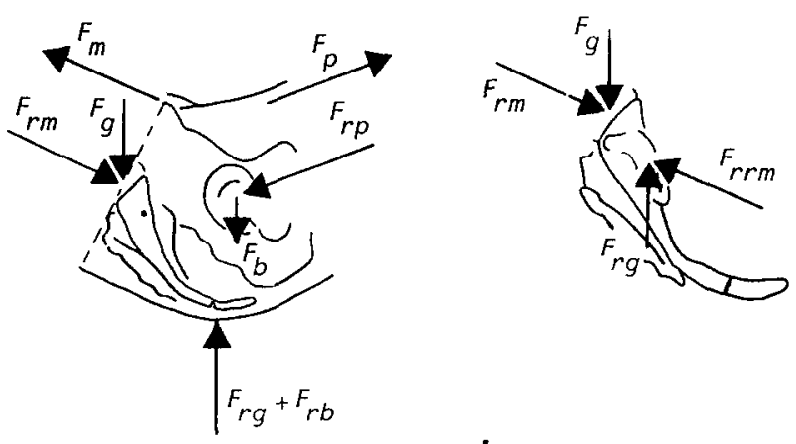

a

b

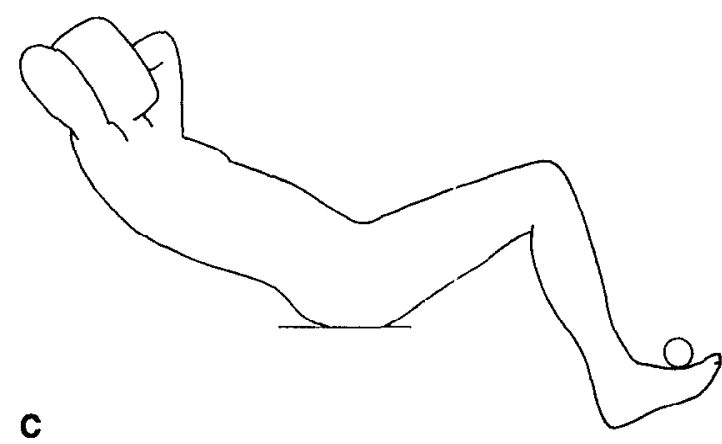

Figure 6. a, Schematic representation of forces acting on the pelvis when exercising abdominal muscles. Abdominal muscles $\left(F_{m}\right)$ and muscles at the ventral side of the hip joints $\left(F_{p}\right)$ provide for equilibrium of the pelvis. The weight of the suprasacral part of the body is $F_{g}$ and $F_{b}$ represents the weight of the pelvis and the legs and the force on the feet. $\mathbf{b}$. Free-body diagram of the sacrum, based on Figure $6 a$. The use of the greater psoas muscle will contribute to $F_{r m}$, which leads to a greater SI joint reaction force $F_{r r m}$.

\section{Discussion}

It is known from the literature ${ }^{1.4}$ that substantial loads are imposed on the lumbar spine when lifting in a stooped posture. Little is known, however, about the transfer of those loads hy the pelvis down to the legs. The focus of our research regards the SI joints in particular. They form discontinuities in large bony structures and are subjected to considerable forces, which emphasize the need of protection against dislocation. In part 1 of this article, we introduced concepts of mechanical principles that can produce the stability required. The second part of this article deals with SI joint loading modes in relation to posture. An $M$ load and an $F$ load were introduced. The $M$ load refers to the forces from muscles that are attached to the sacrum directly or indirectly which produce a moment of force on the sacrum, revealing a tendency to flexion in relation to the hip bones. The $F$ load is the summation of the muscle forces that act on the iliac crests directly, or indirectly by the respective part of the lumbodorsal fascia. The $\mathrm{F}$ loading mode may be appropriate for the transfer of large lcads, because the SI joints can sustain this load with the help of the self-bracing effect of the arch (see part 1), which especially protects against shear. The implementation 
of the lumbodorsal fascia in the process of heavy lifting has becn mentioncd as highly effective for other reasons as well ${ }^{5}$. Part of the lumbodorsal fascia can contribute to the $\mathrm{F}$ load since this part clcarly splits from the spine and remains loose from the sacrum. In anatomical preparations it was shown that between $\mathrm{L}_{5}$ and $S_{1}$ and above the sacrum the fibres of both laminas of the deep fascia cross the midline; they are not attached to the spinous processes nor interspinous ligaments. In some individuals this crossing can even be seen between $\mathrm{L}_{4}$ and $\mathrm{L}_{5}$. This configuration also implies the possibility of, for example, the coupling action of the latissimus dorsi muscle to the action of the heterolateral gluteus maximus muscle.

When the body is in a forward bent posture, the sacrum assumes a more or less horizontal position, and the vertical force from the upper part of the body and the lifted object acts almost perpendicular to the longitudinal axis of the sacrum. This raises the question of whether an effective self-bracing effect can come into action in this plane as well. The contribution required to self-bracing can be expected from the oblique and transverse abdominal muscles. This idea is supported by the observation ${ }^{16}$ that the forces in the direction of the internal and external oblique abdominal muscles, which were manually applied on the anterior superior iliac spines of anatomical preparations, resulted in a close packed position of the SI joints.

The concerted action of muscle groups necessary to perform lifting and trunk flexion/extension tasks is described in the literature. In a recent electromyographic study of the erector spinae, latissimus dorsi, and gluteus maximus during isokinetic lifting experiments, gluteus activity was found to run parallel to latissimus and erector activity ${ }^{18}$. Furthermore, simultaneous acting of erector spinae, hamstrings, and gluteus during trunk bending movements from a standing position has been observed as well as a tendency of the obliquus internus to be active during both trunk flexion and extension ${ }^{19,20}$. These reports on simultaneous activity of hamstrings, gluteus, erector spinae, and abdominal muscles support the concepts in this article, which were developed for the explanation of SI joint stability under load.

\section{Conclusions}

In addition to the biomechanical model in part 1 we can summarize the following conclusions:

- Analysis of loading modes of the SI joint reveals participation of all the muscles that act on the lumbar spine directly or indirectly.

- A bifurcation in the transfer of the resultant back muscle force to the pelvis occurs. This results in two loading modes of the sacrum, which we call the $M$ load and the F load.

- The M load refers to the moment of force about the SI joints produced by the back muscles that partially have an attachment to the sacrum (erector spinae). This can cause a tendency to flexion of the sacrum in relation to the hip bones, which can be counteracted by the dorsal and interosscous sacroiliac, sacrotubcrous, and sacrospinal ligaments. The slightly twisted shape of the SI joint surfaces, resembling the blade of a propeller, can counteract flexion as well, provided the surfaces are pressed together.

- The F load is the summation of the forces of the muscles acting on the iliac bones directly, or indirectly via the lumbodorsal fascia. The $\mathrm{F}$ load, acting in an axial direction to the sacrum, anticipates self-bracing according to the model of the pelvic arch. It was determined that part of the lumbodorsal fascia can contribute to the F load, because this structure clearly splits from the spine and remains loose from the sacrum.

- The larger psoas muscle can also be part of the F load, which follows from a model of an abdominal muscle exercise.

- In a forward bent position the pelvis and consequently the sacrum assume a more or less horizontal position. Therefore, stability of the SI joints requires a self-bracing mechanism in another plane as well. This plane is oriented transverse to the pelvis. We hypothesize that the transverse and oblique abdominal muscles have the proper direction to produce the additional force needed in stooped postures.

- The model on self-bracing can also account for the beneficial effect of the special belts used by weight lifters.

\section{Acknowledgements}

The authors wish to thank M P Bakker, M G van Kruining, R Lip, A L Pool, A H E Slagter, J A C Stigter, and $\mathbf{J} P$ van Wingerden for their valuable contributions.

\section{References}

1 Nachemson A, Elfstrom G. Intravital dynamic pressure measurements in lumbar disc. Scand J Rehabil Med 1970; 1: $1-39$

2 Anderson GBJ, Ortengren R, Schultz AB. Analysis and measurement of the loads on the lumbar spine during work at a table. J Biomech 1980; 13: 513-20

3 Chaffin DB. Biomechanical modelling of the low back during load lifting. Ergonomics 1988; 31: 685-97

4 Schultz AB, Anderson GBJ, Ortengren R et al. Loads on the lumbar spine. J Bone Joint Surg 1982; 64-A: 713-20

5 Gracovetsky S, Farfan HF, Lansy C. The mechanism of the lumbar spine. Spine 1981; 6(3): 249-62

6 Shirazi A. Strain in fibers of a lumbar disc: analysis of the role of lifting in producing disc prolapse. Spine 1989; 14(1): $96-103$

7 Goel VK, Svensson NL. Forces on the pelvis. $J$ Biomech 1977; 10: 195-200

8 Scholten PJM, Schultz AB, Luchies CW, Miller JAA. Motions and loads within the human pelvis: a biomechanical model study. J Orthop Res 1988; 6: 840-50

9 Chandler RF, Clanser CE, McConville JT et al. Investigation of Inertial Properties of the Human Body. Nat Techn Inf Serv 1975; Springfield 
10 Bartelink DL. The role of abdominal pressure in relieving the pressure on the lumbar intervertebral disc. $J$ Bone Joint Surg 1957; 39B: 718

11 Morris M, Lucas DB, Bresler B. Role of the trunk in the stability of the spine. J Bone Joint Surg 1961; A-43A: 327-51

12 Nordin M, Frankel VH. Basic Biomechanics of the Musculoskeletal System. Philadelphia: Lea \& Febiger $1989 ; 142$

13 Vleeming A, Stoeckart R, Snijders CJ. The sacrotuberous ligament: a conceptual approach to its dynamic role in stabilizing the sacro-iliac joint. Clin Biomech 1989; 4: $201-3$

14 Harman EA, Rosenstein RM, Frykman PN, Nigro GA. Effects of a belt on intra-abdominal pressure. Med Sci Sports Exerc 1989; 21(2): 186-90
15 Lander JE, Simonton RL, Giacobbe JK. The effectiveness of weight belts during the squat exercise. Med Sci Sports Exerc 1990; 22(1): 117-26

16 Bojsen-Møller F. Functional anatomy of the sacroiliac joint (in Danish). Månedsskrift for praktisk laegegerning 1988; 4: 211-15

17 Noe DA, Mostardi RA, Jackson ME et al. Myoelectric activity and sequencing of selected trunk muscles during isokinetic lifting. Spine $1992 ; 17(2): 225-9$

18 Oddsson L, Thorstensson A. Fast voluntary trunk flexion movements in standing: motor patterns. Acta Physiol Scand 1987; 129: 93-106

19 Oddsson L, Thorstensson A. Task specificity in the control of intrinsic trunk muscles in man. Acta Physiol Scand 1990; 139: 123-31 